\title{
ALDH1 expression correlates with favorable prognosis in ovarian cancers
}

\author{
Bin Chang ${ }^{1,2,5}$, Guangzhi Liu ${ }^{1,5}$, Fengxia Xue ${ }^{1}$, Daniel G Rosen ${ }^{3}$, Lianchun Xiao ${ }^{4}$, \\ Xuemei Wang ${ }^{4}$ and Jinsong Liu ${ }^{1}$ \\ ${ }^{1}$ Department of Pathology, The University of Texas MD Anderson Cancer Center, Houston, TX, USA; \\ ${ }^{2}$ Department of Pathology/Laboratory of Xinjiang Endemic and Ethnic Diseases, Shihezi University School of \\ Medicine, Shihezi, Xinjiang, China: ${ }^{3}$ Department of Pathology, Baylor College of Medicine, Houston, TX, USA \\ and ${ }^{4}$ Department of Biostatistics, The University of Texas MD Anderson Cancer Center, Houston, TX, USA
}

\begin{abstract}
Aldehyde dehydrogenase 1 (ALDH1), a detoxifying enzyme responsible for the oxidation of intracellular aldehydes, was shown to have a function in the early differentiation of stem cells, through its function in oxidizing retinol to retinoic acid. It has been shown that ALDH1 is a predictor of poor clinical outcome in breast cancer. The authors hypothesized that the level of ALDH1 expression may be correlated with the clinical outcome of patients with ovarian cancer. Immunohistochemical staining of ALDH1 expression was analyzed in 442 primary ovarian carcinomas using tissue microarray. The associations between the expression of the ALDH1 and clinical factors (diagnosis, tumor grade, stage, and clinical response to chemotherapy), as well as overall and disease-free survival, were analyzed. Expression of ALDH1 was found in $48.9 \%$ of the samples. Fisher's exact test suggested that high expression of ALDH1 was significantly associated with endometrioid adenocarcinoma $(P<0.0001)$, early-stage disease $(P=0.006)$, complete response to chemotherapy $(P<0.05)$, and a low serum level of CA125 $(P=0.02)$. High percentage of cells expressing ALDH1 was associated with a longer overall survival time $(P=0.01)$ and disease-free survival time $(P=0.006)$ by log-rank test. In contrast to its function in breast cancer, ALDH1 was a favorable prognostic factor in ovarian carcinoma. ALDH1 therefore may have a different function in ovarian cancer than it does in breast cancer.
\end{abstract}

Modern Pathology (2009) 22, 817-823; doi:10.1038/modpathol.2009.35; published online 27 March 2009

Keywords: ALDH1; ovarian cancer; immunohistochemistry; prognosis

Aldehyde dehydrogenase (ALDH) is a family of ubiquitous enzymes located in nearly all mammalian tissues, which catalyze the oxidation of aldehydes to their carboxylic acid forms. ${ }^{1,2}$ They participate in the detoxification of acetaldehyde, ${ }^{1,2}$ the metabolism of biogenic amines, ${ }^{3}$ corticosteroids, ${ }^{4}$ and retinoic acid..$^{5,6}$

To date, 17 isoforms of ALDH had been described. ${ }^{7}$ ALDH1 is the cytosolic isoform. Murine and human hematopoietic stem/progenitor cells have been isolated based on their high levels of ALDH activity. ${ }^{8-13}$ ALDH activity has been used to identify stem-like subsets not only in human hematopoietic cancers but also in solid cancers

Correspondence: Dr J Liu, MD, PhD, Department of Pathology, Unit 85, The University of Texas MD Anderson Cancer Center, 1515 Holcombe Boulevard, Houston, TX 77030, USA.

E-mail: jliu@mdanderson.org

${ }^{5}$ These authors contributed equally to this work.

Received 17 December 2008; revised and accepted 17 February 2009; published online 27 March 2009 (eg, breast cancer). ${ }^{14-17}$ A recent study by Ginestier et $a l^{15}$ demonstrated that high ALDH activity selected for both normal and tumorigenic human mammary epithelial cells with stem/progenitor properties, expression of ALDH1 was a predictor of poor clinical outcome in breast tumors. However, it remains controversial that these cell-surface markers can be used as the sole means to isolate cancer stem cells because of the heterogeneous nature of solid tumors. ${ }^{14,18-22}$ Furthermore, the clinical, pathologi$\mathrm{cal}$, and immunohistochemical features of cancers associated with high expression of these markers remain unknown.

Ovarian cancer ranks as the fifth leading cause of cancer deaths among women. ${ }^{23}$ The expression of ALDH1 and its clinical significance is unknown in ovarian cancer. The authors hypothesized that the level of ALDH1 expression may be correlated with the clinical outcome of patients with ovarian cancer. The purpose of this study therefore was to evaluate the association between the expression of ALDH1 and the clinical pathological factors (including 
diagnosis, tumor grade, disease stage, and clinical response to chemotherapy), as well as the overall survival and disease-free survival of ovarian cancer. To do this, we retrospectively analyzed the clinical pathological factors of 442 cases of primary ovarian cancer and subjected archived tissue specimens to microarray analysis of the expression of putative cancer stem cell marker ALDH1 by immunohistochemical staining and correlate with the clinical outcome.

\section{Materials and methods}

\section{Patients and Clinicopathologic Data}

This study analyzed tumor samples from women diagnosed with primary ovarian carcinoma that had undergone initial surgery at The University of Texas MD Anderson Cancer Center between 1 January 1990 and 31 December 2005. Depending on the availability of representative tumor samples, we obtained data for 442 patients. The relevant clinical data were collected by retrospective review of the patients' files. Follow-up information was updated through May 2008 by reviewing medical records and the United States Social Security Index. Histopathologic diagnoses were based on World Health Organization criteria, ${ }^{24}$ tumor grading for non-serous carcinomas was based on Gynecologic Oncology Group criteria, ${ }^{25-27}$ and disease staging was assigned according to the International Federation of Gynecology and Obstetrics staging system. $^{28}$ Serous carcinomas were graded by using a two-tier system (low grade and high grade) according to the criteria proposed by Malpica et al. ${ }^{29}$

To analyze response to primary therapy, we classified patients as responders or nonresponders. Patients who entered complete clinical remission with a normal CA125 level after chemotherapy for histological or cytological diagnosis of ovarian carcinoma with a treatment-free interval of $\geq 6$ months were defined as responders. ${ }^{30}$

The group of nonresponders also was subdivided according to whether patients had progressive disease or recurrent disease. Progressive disease was defined as progression that occurred without disease remission observed after the initiation of treatment; recurrent disease was defined as disease that was detected after a period of clinically documented remission that was not sustained. ${ }^{31,32}$

\section{Construction of the Tissue Microarrays}

We constructed tissue microarray blocks by taking one representative paraffin-embedded block from every patient and taking one core from morphologically representative areas of blocks as previously described. $^{33}$ The use of tissue blocks and chart reviews was approved by the institutional review board of The University of Texas MD Anderson Cancer Center.

\section{Immunohistochemical Analysis}

Tissue microarray slides were subjected to immunohistochemical staining according to the manufacturer's protocol (Biocare Medical, Concord, CA, USA). In brief, after initial deparaffinization/hydration, sections were microwaved for $15 \mathrm{~min}$ in $10 \mathrm{mM}$ citrate buffer, $\mathrm{pH}$ 6.0, to unmask the epitopes. Endogenous peroxidase activity was blocked by using $3 \%$ hydrogen peroxide. Nonspecific binding was blocked with background sniper (Biocare Medical) and slides were incubated for $10 \mathrm{~min}$ at room temperature. The slides were then incubated overnight at $4^{\circ} \mathrm{C}$ with primary mouse monoclonal antibody against ALDH1 (clone 44/ALDH, 1:100 dilution; BD Biosciences, San Jose, CA, USA); with a biotin-labeled secondary antibody (Universal Goat Link; Biocare Medical) for $15 \mathrm{~min}$; and finally with HRP (Biocare Medical) for $15 \mathrm{~min}$. Tissues were then stained for $5 \mathrm{~min}$ with $3,3^{\prime}$ diaminobenzidine (Biocare Medical). Finally, tissues were counterstained with hematoxylin, dehydrated, and mounted in DePex. Negative controls were made by replacing the primary antibody with phosphate-buffered saline. The intensely ALDH1positive stromal cells were used as internal positive controls.

Immunohistochemical stainings for ALDH1 were analyzed by two gynecological pathologists (JL and BC). Staining scored only according to cytoplasmic staining of the cancer cells. The degree of staining was quantified using a four-score grading system. Cores with $<5 \%$ ALDH1-positive cells were given a score of 0 , those with 5-20\% ALDH1positive cells were given a score of 1 , those with 20-50\% ALDH1-positive cells were scored as 2, and those with $>50 \%$ ALDH1-positive cells were scored as 3 . For the statistical analysis, we divided cases into two groups: low expression (with scores of 0 or 1 ) and high expression (with scores of 2 or 3 ).

\section{Statistical Analysis}

Fisher's exact test and logistic regression analysis were performed to evaluate the association of ALDH1 with clinical factors. The Kaplan-Meier method was used to estimate the probability of overall survival and disease-free survival, and the log-rank test was used to compare the overall survival or disease-free survival between different comparison groups, such as patients with low or high ALDH1 expressions. Multivariate Cox proportional hazards regression models were fitted to determine the significant factors associated with overall survival and disease-free survival and to assess the association of ALDH1 with overall 
survival or disease-free survival after adjusting the effect of other clinical factors. The overall survival time was computed as the time interval from the date of first biopsy to the date of death or the last follow-up date, whichever occurred first. Patients alive on the last follow-up date were censored. The disease survival time was computed as the time period from the date of first biopsy to the date of recurrence, the date of death, or the date of last follow-up, whichever occurred first. Patients alive on the last follow-up date without recurrence were censored. Results were considered statistically significant at the $P<0.05$ level. SAS 9.1 software (SAS Institute Inc., Cary, NC, USA) was used for the statistical analysis.

\section{Results}

\section{Patient Characteristics}

The median age of the 442 patients was 60 years (range, 21-89 years). The median overall survival time was 4.0 years (95\% CI: $3.5-4.6$ years), and the overall survival rates were $60 \%$ (95\% CI: $0.55-0.65$ ) at 3 years, $42 \%$ (95\% CI: $0.37-0.47$ ) at 5 years, and $27 \%(0.22-0.32)$ at 10 years. The median follow-up interval was 8.0 years with a 95\% confidence interval of 6.5-10.2 years.

\section{ALDH1 Expression and Localization}

Diffuse cytoplasmic staining with moderate intensity was observed in different proportion of the tumor cells. Very strong cytoplasmic and nuclear staining was observed in stromal cells. The expression in epithelial cancer cells was scored and subjected for statistical analysis. The percentage of positive cancer cells varied from $<5$ to $>50 \%$ in our patient population (Figure 1).

\section{Association Between Expression of ALDH1 and Clinicopathologic Variables}

The results of immunostaining of the tumor microarrays, organized according to clinicopathologic characteristics of the patients, are shown in Table 1. No ALDH1 was expressed $($ score $=0)$ in 226 patients (51\%), $1-5 \%$ of the cells expressed ALDH1 in 82 patients (19\%; score also $=0), 6-20 \%$ of the cells expressed ALDH1 in 49 patients $(11 \%$; score $=1), 21-50 \%$ of the cells expressed ALDH1 in 46 patients $(10 \%$; score $=2)$, and $>50 \%$ of cells expressed ALDH1 in 41 patients $(9 \%$; score $=3)$. High expression of ALDH1 ( $>20 \%$ of cells) was associated with endometrioid adenocarcinoma $(P<0.0001)$, early-stage disease $(P=0.006)$ (the cutoffs for early vs late stage is I vs II-IV), and low
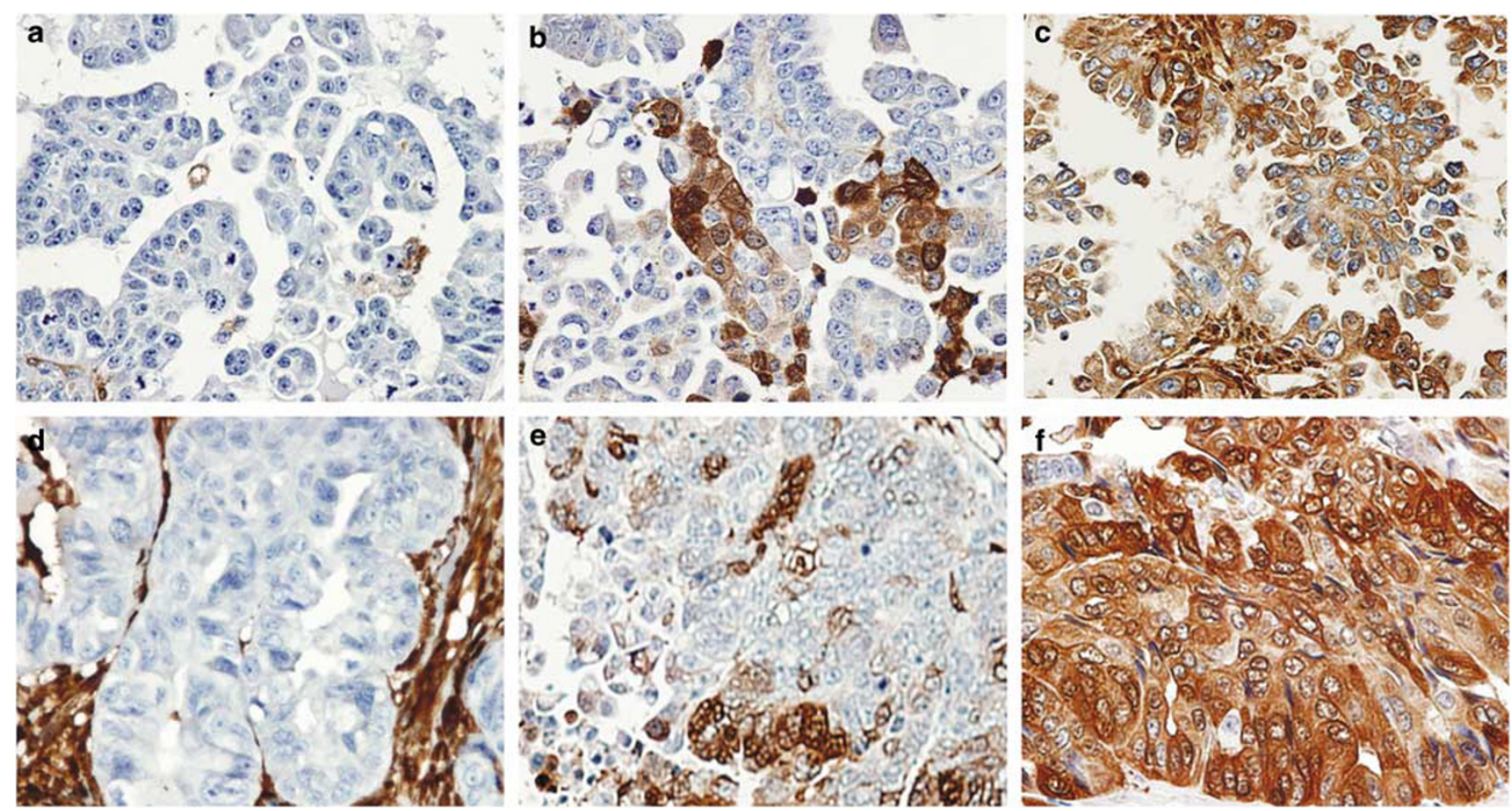

Figure 1 Immunoreactivity patterns of ALDH1 in ovarian serous adenocarcinomas (a-c) and endometrioid adenocarcinomas (d-f). (a) ALDH1-negative staining in serous carcinoma. (b) Serous carcinoma cells $(\leq 20 \%)$ show cytoplasmic staining for ALDH1. (c) Diffuse positive staining for ALDH1 in serous carcinoma. (d) ALDH1-negative staining in endometrioid adenocarcinoma. (e) Endometrioid carcinoma cells $(\leq 20 \%)$ show cytoplasmic staining for ALDH1. (f) Diffused positive staining for ALDH1 in endometrioid carcinoma (original magnification $\times 200$ ). 
Table 1 Correlations between the expression of ALDH and clinicopathologic factors

\begin{tabular}{|c|c|c|c|c|}
\hline \multirow[t]{2}{*}{ Characteristic } & \multicolumn{3}{|c|}{ No. of patients (\%) } & \multirow[b]{2}{*}{$\mathrm{P}^{*}$} \\
\hline & $\leq 20 \%$ ALDH-positive cells & $>20 \%$ ALDH-positive cells & Total no. & \\
\hline Histologic type & & & & $<0.0001$ \\
\hline Serous carcinoma & $227(85)$ & $39(15)$ & 266 & \\
\hline Endometrioid carcinoma & $15(43)$ & $20(57)$ & 35 & \\
\hline Mucinous carcinoma & $3(60)$ & $2(40)$ & 5 & \\
\hline Clear-cell carcinoma & $12(86)$ & $2(14)$ & 14 & \\
\hline MMMT & $12(70)$ & $5(30)$ & 17 & \\
\hline Poorly differentiated carcinoma & 7 (88) & $1(12)$ & 8 & \\
\hline Transitional cell carcinoma & $6(100)$ & $0(0)$ & 6 & \\
\hline Mixed-type carcinoma & $75(82)$ & $16(18)$ & 91 & \\
\hline FIGO disease stage & & & & 0.006 \\
\hline Stage I & $18(56)$ & $14(44)$ & 32 & \\
\hline Stage II & $24(80)$ & $6(20)$ & 30 & \\
\hline Stage III & $250(82)$ & $55(18)$ & 305 & \\
\hline Stage IV & $60(83)$ & $12(17)$ & 72 & \\
\hline Unknown & & & 3 & \\
\hline \multicolumn{5}{|l|}{ CA125 (U/ml) } \\
\hline$<500$ & $55(71)$ & $23(29)$ & 78 & 0.02 \\
\hline$\geq 500$ & $113(84)$ & $22(16)$ & 135 & \\
\hline Unknown & & & 229 & \\
\hline
\end{tabular}

FIGO, International Federation of Gynecology and Obstetrics; MMMT, malignant mixed Mullerian tumor.

${ }^{*} P$-values were calculated by Fisher's exact test.

serum CA125 level $(P=0.02)$. We analyzed the correlation between the expression of ALDH1 and the grade of serous carcinoma and endometrioid carcinoma separately because of different grading systems used in serous carcinoma and endometrioid carcinoma. No correlation was found between the expression of the ALDH1 and grade in either the serous or endometrioid carcinoma (data not shown).

The correlation of ALDH1 expression (high or low) with response to primary therapy is shown in Table 2. In total, 346 patients $(78 \%)$ received postsurgical cisplatin-based treatment, either alone or in combination with other adjuvant drugs. In 46 patients $(10 \%)$, cisplatin-based treatment was administered before surgical debulking surgery. Three patients $(1 \%)$ received other forms of treatment (melphalan, 5-fluorouracil plus folinic acid). In 5 patients $(1 \%)$, the treatment protocol was unknown. Overall, higher levels of expression of ALDH1 $(>20 \%)$ were observed in the complete response group than in nonresponse group $(P=0.0002)$. Similar proportions of ALDH1 expression were observed in both the postsurgical cisplatin-based treatment subgroup $(P=0.01$ and the presurgical cisplatin-based treatment subgroup $(P=0.04)$.

The multivariate logistic regression analysis suggested that ALDH1 was significantly associated with histological type and response to chemotherapy (see Supplementary Table 1). Patients with endometrioid carcinoma would be more likely to have a higher ALDH1 expression ( $>20 \%$ ) compared with those with serous adenocarcinoma $(\mathrm{OR}=6.12, P<0.0001)$.
Patients with progression disease and recurrent disease would be less likely to have a higher ALDH1 expression $(\mathrm{OR}=0.31,0.45$ and $P=0.03$ and 0.03 , respectively).

\section{Association of ALDH1 with Overall Survival and Disease-Free Survival}

Overall survival and disease-free survival rates at 3, 5 , and 10 years are shown in relation to the expression of ALDH1 in Tables 3 and 4 . At the time of this report, 88 of the 442 analyzed patients were alive without clinical evidence of ovarian carcinoma, 75 were alive with ovarian carcinoma, 262 had died of ovarian carcinoma, 15 were alive with unknown ovarian carcinoma status, and 2 had been lost to follow-up who were excluded from the overall survival and disease-free survival analysis. ALDH1 expression correlated with overall survival as well as disease-free survival. Patients who had tumors with $>20 \%$ ALDH1-positive cells had better overall survival rate $(P=0.01)$ and disease-free survival rate $(P=0.006)$ than patients who had tumors with $<20 \%$ ALDH1-positive cells (Figure 2).

The multivariate Cox proportional hazards regression analysis indicated that stage was significantly associated with overall survival and disease-free survival. Patients with stage II, III, and IV would have a higher risk to be dead compared to stage I patients $(\mathrm{HR}=4.32,7.2,9.6$ and $P=0.01,0.0002$, 0.00002 , respectively). Patients with stage II, III, and IV would have a higher risk of event (ie, recurrence, 
progression, or death) compared to stage I patients $(\mathrm{HR}=2.4,5.7,7.3$ and $P=0.048,<0.0001,<0.0001$, respectively).

Table 2 Correlation of ALDH1 expression and response to primary therapy

\begin{tabular}{|c|c|c|c|}
\hline \multirow{2}{*}{$\begin{array}{l}\text { Response to primary } \\
\text { therapy* }\end{array}$} & \multicolumn{3}{|c|}{ No. of patients } \\
\hline & $\begin{array}{l}\leq 20 \% \text { ALDH1- } \\
\text { positive cells }\end{array}$ & $\begin{array}{l}>20 \% \text { ALDH- } \\
\text { positive cells }\end{array}$ & Total \\
\hline Unknown response & 10 & 2 & 12 \\
\hline \multicolumn{4}{|l|}{ Responders } \\
\hline \multicolumn{4}{|c|}{ Cisplatin-based regimens } \\
\hline Postsurgery & 170 & 53 & 223 \\
\hline Presurgery ${ }^{\mathrm{b}}$ & 9 & 6 & 15 \\
\hline Other regimens & 0 & 0 & 0 \\
\hline Unknown regimen & 3 & 0 & 3 \\
\hline \multicolumn{4}{|c|}{ Nonresponders, progressive disease } \\
\hline \multicolumn{4}{|c|}{ Cisplatin-based regimens } \\
\hline Postsurgery ${ }^{\mathrm{a}}$ & 84 & 11 & 95 \\
\hline Presurgery & 21 & 4 & 25 \\
\hline Other regimens & 1 & 0 & 1 \\
\hline Unknown regimen & 1 & 0 & 1 \\
\hline \multicolumn{4}{|c|}{ Nonresponders, recurrent disease } \\
\hline \multicolumn{4}{|c|}{ Cisplatin-based regimens } \\
\hline Postsurgery $^{\mathrm{a}}$ & 26 & 2 & 28 \\
\hline Presurgery $^{\mathrm{b}}$ & 6 & 0 & 6 \\
\hline Other regimens & 2 & 0 & 2 \\
\hline Unknown regimen & 1 & 0 & 1 \\
\hline No chemotherapy & 11 & 9 & 20 \\
\hline Total & 321 & 121 & 442 \\
\hline
\end{tabular}

${ }^{\mathrm{a}}$ Cisplatin-based postsurgery subgroup $(P=0.01)$.

${ }^{\mathrm{b}}$ Cisplatin-based presurgery subgroup $(P=0.04)$.

${ }^{*} P$-values were calculated by using Fisher's exact test (response to primary therapy, $P=0.0002$ ).

\section{Discussion}

In the current study of 442 well-characterized patients with long-term follow-up, high levels of ALDH1 expression were observed in $19 \%$ of the ovarian carcinoma samples, which correlated with endometrioid adenocarcinoma, early- stage disease, complete response to chemotherapy, and low serum CA125 level and favorable survival. In our study, multivariate Cox proportional hazards regression analysis showed that the early stage of disease was strongly associated with longer overall survival and disease-free survival $(P<0.0002$ and $P<0.0001$, respectively) and was a independent prognosis predicator. Although ALDH1 was not an independent predictor in multivariate analysis, high expression of ALDH1 was associated with early-stage disease $(P=0.006)$. It gave us a clue that ALDH1 might be a potential-independent prognosis predictor in ovarian cancer. Our results are contrast with the report by Ginestier et $a l^{15}$ who demonstrated that the expression of ALDH1 was an independent predictor of poor clinical outcome in breast tumors. We found that high expression of ALDH1 was associated longer overall survival in patients with ovarian carcinoma. ALDH1 may have a different function in ovarian cancer than it does in breast cancer.

ALDH1 has been demonstrated to be a stem cell marker in several types of malignancies. ${ }^{14-17}$ Theoretically, a high proportion of cancer stem cells in the tumor should be correlated with a poor prognosis. However, depending on the cancer site, markers used to identify stem cells from one organ may or may not be useful for identifying stem cells from other organs or tumor types. ${ }^{18,34,35}$ Our result, which is based on a large body of clinical material, demonstrates that expression of ALDH1 is correlated with favorable clinical outcome in patients

Table 3 ALDH1 expression and overall survival

\begin{tabular}{|c|c|c|c|c|c|c|}
\hline \multirow[t]{2}{*}{ ALDH expression } & \multirow[t]{2}{*}{ No. of patients } & \multirow[t]{2}{*}{ Median survival (95\% CI) years } & \multicolumn{3}{|c|}{ Survival rate (95\% CI) } & \multirow[b]{2}{*}{$\mathrm{P}^{*}$} \\
\hline & & & 3-year & 5-year & 10-year & \\
\hline $\begin{array}{l}\leq 20 \% \\
>20 \%\end{array}$ & $\begin{array}{r}353 \\
87\end{array}$ & $\begin{array}{l}3.8(3.2,4.4) \\
5.8(3.5, N A)\end{array}$ & $0.58(0.53,0.64)$ & $0.39(0.33,0.45)$ & $0.23(0.18,0.29)$ & 0.01 \\
\hline
\end{tabular}

${ }^{*} P$-values were derived from the log-rank test.

Table 4 ALDH1 expression and disease-free survival

\begin{tabular}{|c|c|c|c|c|c|c|}
\hline \multirow[t]{2}{*}{$A L D H$ expression } & \multirow{2}{*}{ No. of patients } & \multirow{2}{*}{ Median survival ( $95 \%$ CI) years } & \multicolumn{3}{|c|}{ Survival rate $(95 \%$ CI) } & \multirow[b]{2}{*}{$\mathrm{P}^{*}$} \\
\hline & & & 3-year & 5-year & 10-year & \\
\hline$\leq 20 \%$ & 353 & $3.1(2.8,3.7)$ & $0.52(0.47,0.57)$ & $0.29(0.24,0.34)$ & $0.13(0.1,0.18)$ & 0.006 \\
\hline$>20 \%$ & 87 & $4.4(3.3,5.8)$ & $0.64(0.54,0.75$ & $0.44(0.34,0.56)$ & $0.29(0.2,0.42)$ & \\
\hline
\end{tabular}

${ }^{*} P$-values were derived from the log-rank test. 

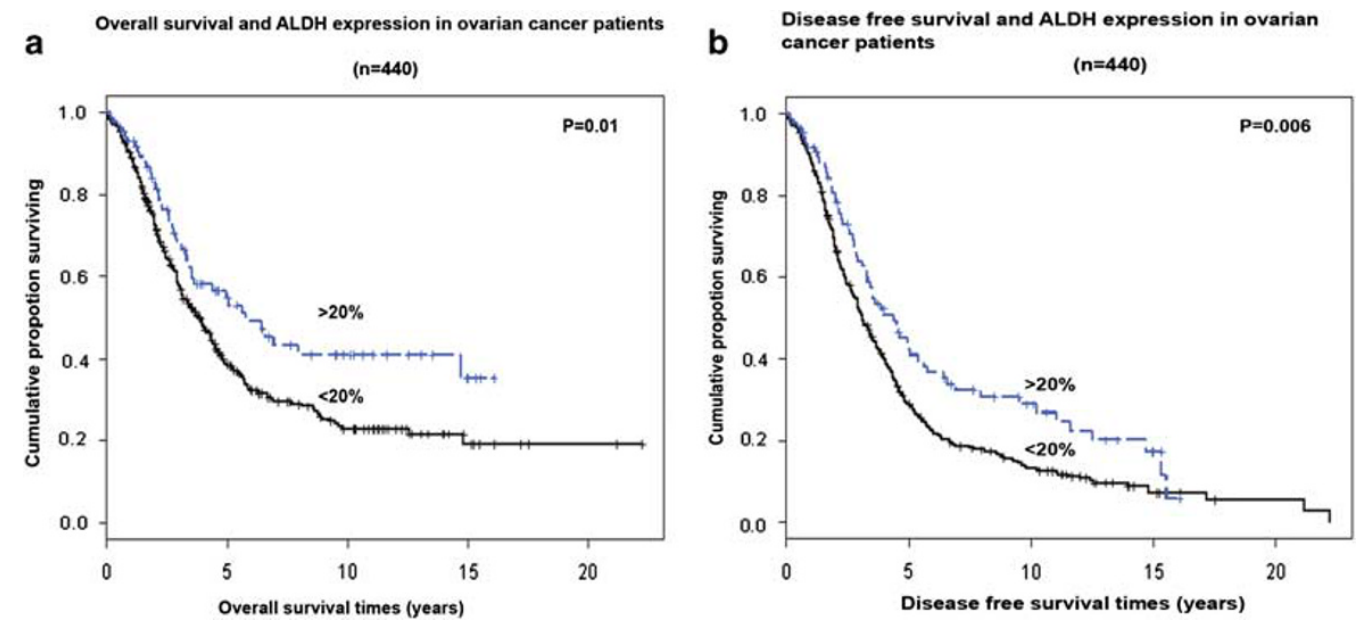

Figure 2 Kaplan-Meier survival curves for groups of ovarian carcinoma patients with low and high levels of ALDH1 expression. (a) Overall survival curves in all patients with ovarian cancer $(n=440)$. (b) Disease-free survival curves in all patients $(n=440)$.

with ovarian carcinoma. It remains to be determined whether ALDH1 is associated with a stem cell or stem-like cells in human ovarian cancer.

In summary, the current results demonstrate that in contrast to its function in breast cancer, ALDH1 has a favorable function in ovarian carcinoma and thus high expression of ALDH1 is a favorable prognostic factor in patients with ovarian cancer. ALDH1 thus may have a different function in ovarian cancer than it does in breast cancer.

\section{Acknowledgements}

We thank Dr Gong Yang, Imelda Mercado-Uribe, and Xue Xiao for their helpful discussions regarding the study design. We appreciate Kate J Newberry for her helpful editing for the paper. JL was supported by a Research Scholar Grant (RSG-04-028-1-CCE) from the American Cancer Society and by R01 CA13118301 from the National Institutes of Health/National Cancer Institute (NIH/NCI), and Institutional Research Grant.

\section{Disclosure/conflict of interest}

No potential conflicts of interest were disclosed.

\section{References}

1 Lindahl R. Aldehyde dehydrogenases and their role in carcinogenesis. Crit Rev Biochem Mol Biol 1992;27: 283-335.

2 Deitrich RA, Petersen D, Vasiliou V. Removal of acetaldehyde from the body. Novartis Found Symp 2007;285:23-40.

3 Ambroziak W, Pietruszko R. Human aldehyde dehydrogenase. Activity with aldehyde metabolites of monoamines, diamines, and polyamines. J Biol Chem 1991;266:13011-13018.

4 Monder C, Purkaystha AR, Pietruszko R. Oxidation of the 17-aldol ( 20 beta hydroxy-21-aldehyde) intermediate of corticosteroid metabolism to hydroxy acids by homogeneous human liver aldehyde dehydrogenases. J Steroid Biochem 1982;17:41-49.

5 Duester G, Mic FA, Molotkov A. Cytosolic retinoid dehydrogenases govern ubiquitous metabolism of retinol to retinaldehyde followed by tissue-specific metabolism to retinoic acid. Chem Biol Interact 2003;143-144:201-210.

6 Ambroziak W, Izaguirre G, Pietruszko R. Metabolism of retinaldehyde and other aldehydes in soluble extracts of human liver and kidney. J Biol Chem 1999;274: 33366-33373.

7 Sladek NE. Human aldehyde dehydrogenases: potential pathological, pharmacological, and toxicological impact. J Biochem Mol Toxicol 2003;17:7-23.

8 Kastan MB, Schlaffer E, Russo JE, et al. Direct demonstration of elevated aldehyde dehydrogenase in human hematopoietic progenitor cells. Blood 1990; 75:1947-1950.

9 Storms RW, Trujillo AP, Springer JB, et al. Isolation of primitive human hematopoietic progenitors on the basis of aldehyde dehydrogenase activity. Proc Natl Acad Sci USA 1999;96:9118-9123.

10 Hess DA, Wirthlin L, Craft TP, et al. Selection based on CD133 and high aldehyde dehydrogenase activity isolates long-term reconstituting human hematopoietic stem cells. Blood 2006;107:2162-2169.

11 Cai J, Cheng A, Luo Y, et al. Membrane properties of rat embryonic multipotent neural stem cells. J Neurochem 2004;88:212-226.

12 Hess DA, Meyerrose TE, Wirthlin L, et al. Functional characterization of highly purified human hematopoietic repopulating cells isolated according to aldehyde dehydrogenase activity. Blood 2004;104: 1648-1655.

13 Armstrong L, Stojkovic M, Dimmick I, et al. Phenotypic characterization of murine primitive hematopoietic progenitor cells isolated on basis of aldehyde dehydrogenase activity. Stem Cells 2004;22: 1142-1151. 
14 Croker AK, Goodale D, Chu J, et al. High aldehyde dehydrogenase and expression of cancer stem cell markers selects for breast cancer cells with enhanced malignant and metastatic ability. J Cell Mol Med; 4 August 2008 (e-pub ahead of print).

15 Ginestier C, Hur MH, Charafe-Jauffret E, et al. ALDH1 is a marker of normal and malignant human mammary stem cells and a predictor of poor clinical outcome. Cell Stem Cell 2007;1:555-567.

16 Pearce DJ, Taussig D, Simpson C, et al. Characterization of cells with a high aldehyde dehydrogenase activity from cord blood and acute myeloid leukemia samples. Stem Cells 2005;23:752-760.

17 Ma S, Chan KW, Lee TK, et al. Aldehyde dehydrogenase discriminates the CD133 liver cancer stem cell populations. Mol Cancer Res 2008;6:1146-1153.

18 Clarke MF, Dick JE, Dirks PB, et al. Cancer stem cellsperspectives on current status and future directions: AACR workshop on cancer stem cells. Cancer Res 2006;66:9339-9344.

19 Polyak K. Breast cancer stem cells: a case of mistaken identity? Stem Cell Rev 2007;3:107-109.

20 Fillmore C, Kuperwasser C. Human breast cancer stem cell markers CD44 and CD24: enriching for cells with functional properties in mice or in man? Breast Cancer Res 2007;9:303.

21 Shipitsin M, Campbell LL, Argani P, et al. Molecular definition of breast tumor heterogeneity. Cancer Cell 2007;11:259-273.

22 Levi BP, Yilmaz OH, Duester G, et al. Aldehyde dehydrogenase 1a1 is dispensable for stem cell function in the mouse hematopoietic and nervous systems. Blood 2009;113:1670-1680.

23 Jemal A, Siegel R, Ward E, et al. Cancer statistics, 2008. CA Cancer J Clin 2008;58:71-96.

24 Scully RE. World Health Organization classification and nomenclature of ovarian cancer. Natl Cancer Inst Monogr 1975;42:5-7.
25 Russell P. The pathological assessment of ovarian neoplasms. I: introduction to the common 'epithelial' tumours and analysis of benign 'epithelial' tumours. Pathology 1979;11:5-26.

26 Russell P. The pathological assessment of ovarian neoplasms. II: the proliferating 'epithelial' tumours. Pathology 1979;11:251-282.

27 Russell P. The pathological assessment of ovarian neoplasms. III: the malignant 'epithelial' tumours. Pathology 1979;11:493-532.

28 Shepherd JH. Revised FIGO staging for gynaecological cancer. Br J Obstet Gynaecol 1989;96:889-892.

29 Malpica A, Deavers MT, Lu K, et al. Grading ovarian serous carcinoma using a two-tier system. Am J Surg Pathol 2004;28:496-504.

30 Rustin GJ, Nelstrop AE, Crawford M, et al. Phase II trial of oral altretamine for relapsed ovarian carcinoma: evaluation of defining response by serum CA125. J Clin Oncol 1997;15:172-176.

31 Armstrong DK. Relapsed ovarian cancer: challenges and management strategies for a chronic disease. Oncologist 2002;7(Suppl 5):20-28.

32 Rustin GJ, Nelstrop AE, Tuxen MK, et al. Defining progression of ovarian carcinoma during follow-up according to CA 125: a North Thames Ovary Group Study. Ann Oncol 1996;7:361-364.

33 Rosen DG, Yang G, Deavers MT, et al. Cyclin E expression is correlated with tumor progression and predicts a poor prognosis in patients with ovarian carcinoma. Cancer 2006;106:1925-1932.

34 Bradfute SB, Graubert TA, Goodell MA. Roles of Sca-1 in hematopoietic stem/progenitor cell function. Exp Hematol 2005;33:836-843.

35 Burger PE, Xiong X, Coetzee S, et al. Sca-1 expression identifies stem cells in the proximal region of prostatic ducts with high capacity to reconstitute prostatic tissue. Proc Natl Acad Sci USA 2005;102 $7180-7185$.

Supplementary Information accompanies the paper on Modern Pathology website (http://www.nature.com/ modpathol) 